\title{
News Communication Value Evaluation Algorithm Based on Deep Neural Network
}

\author{
Di Zhang \\ New Media Communication Department, School of Humanities, Jiamusi University, Jiamusi 154002, China \\ Correspondence should be addressed to Di Zhang; zhangdi@jmsu.edu.cn
}

Received 14 November 2021; Revised 15 December 2021; Accepted 16 December 2021; Published 22 January 2022

Academic Editor: Xin Ning

Copyright (c) 2022 Di Zhang. This is an open access article distributed under the Creative Commons Attribution License, which permits unrestricted use, distribution, and reproduction in any medium, provided the original work is properly cited.

\begin{abstract}
The evaluation of the value of news communication is an area that deserves more attention, but there has been a dearth of specific research in this area. We must first determine the positioning of the news dissemination activity in order to study the mechanism for evaluating the effect of news dissemination. Fundamentally, assessing the impact of news dissemination is a dialectical balance of accuracy and effectiveness. I can conduct research from the three dimensions of policy orientation, communication orientation, and audience orientation and scientifically evaluate the effect of news communication on a qualitative and quantitative basis, based on the main body of the publisher. With only a small amount of labelled data, a high-quality news propagation value evaluation can be achieved. The value is rationally searched in the era of algorithm recommendation. The traditional "personalised recommendation algorithm" caters to the audience's interests in one direction, with the goal of gaining their attention and commercial success. The "personalised recommendation algorithm" primarily uses statistical methods in the processing of news information, such as user's basic information and behaviour information, and does not investigate the attributes and functions of news facts or the quality of news information itself. The research proposed in this paper suggests attempting to design an algorithm based on "news value." First, it is based on the premise of a rational and caring "public person" who is concerned about the public interest, as well as professional journalistic news selection criteria. "News value" is a theoretical foundation for algorithm design that considers not only the publicity of news but also the audience's interests. More importantly, this type of algorithm is designed to judge the quality and value of information based on how well the information is understood and then make a decision, returning to the essence of news facts and information.
\end{abstract}

\section{Introduction}

The recommendation system has the ability to solve information overload and mine customer data and has been widely used. In the current press release research, the effect evaluation mechanism has always been an area worthy of attention, but there is relatively a lack of specific research [1]. On the one hand, the news release activity itself is also a communication activity, and it is also suitable for the attention of the communication effect research mechanism [2]. Therefore, in many communication effect research works, the effect evaluation of political communication activities will involve the effect of news release [3]. But, from another perspective, press release is a relatively independent government information activity, and the construction of mechanisms involved in it all has an impact on the information dissemination link $[4,5]$. However, although the number of domestic journalists' research results on the theory of news value has been increasing since the new era, the degree of theoretical consensus among scholars is very low.

This paper aims to build a communication effect evaluation system for news communication value evaluation using the deep neural network algorithm. The evaluation system has four components: an evaluation subject, an evaluation object, an evaluation standard, and an evaluation method [6]. Those organisations or individuals who are assessing the impact of the press conference are referred to as the assessment subject. Media, audiences, information publishers, and published information are all examples of evaluation objects [7]. The deep neural network algorithmbased evaluation criteria are divided into two levels. The first 
is a first-level evaluation index that includes mass media, audience, news spokesperson, and information as its core components. The other factors are the number of reports, report attitude, audience composition, and agenda setting [8]. The core secondary evaluation indicators are information accuracy, information timeliness, and so on.

\section{Related Works}

The ultimate pursuit of any news dissemination value activity is to obtain a good dissemination effect. Therefore, whether it is from the academic level or from the operational level [9], it is particularly important to study how to expand the effect of news dissemination [10]. News has a complete and orderly information delivery system.

The document model is a new CTR prediction model jointly proposed by Huawei Noah's Ark Laboratory and Harbin Institute of Technology in 2017. This model effectively combines the advantages of factorization machines and neural networks in feature learning and can extract lowlevel combined features at the same time and combine features with high-level features, and no feature engineering is required besides original features. However, the hyperparameters of the number of hidden layers and the number of neurons in each layer cannot be accurately set, resulting in a decrease in its prediction accuracy.

Literature [11] proposes that the communication effect is divided into three levels in communication science [12]. According to the logical sequence or performance stage of its occurrence, it can be divided into external information acting on people's intuition and memory system causing an increase in people's knowledge and a change in knowledge composition [13]. The effects on the cognitive level act on people's ideas or value systems to cause changes in emotions or feelings, which are effects on the psychological and attitude levels $[14,15]$. These changes are manifested by people's words and deeds, which become effects on the behaviour level. From cognition to attitude to action is a process of accumulation, deepening, and expansion of effects.

Years of social propaganda practical experience show that different propagandists have different propaganda effects when propagating the same content of information, according to the literature [16]. The main reason is that propagandists have varying degrees of credibility. The credibility of the propagandist is made up of two elements: the propagator's credibility and professional authority [17]. The "credibility effect," as defined by Hofland, stated that the higher the source's credibility, the greater the persuasive effect; the lower the source's credibility, the smaller the persuasive effect. The primary cause of this phenomenon is that people with a good reputation and authority are recognised and praised by society. Their behaviour patterns are frequently models for the audience, and the information they release is readily accepted and recognised. According to literature [18], in the field of news dissemination, algorithms' classification [19-21], retrieval, and dissemination of information may inadvertently become accomplices of certain social prejudices, copying racial discrimination or prejudice that already exists in society.

Literature [22] proposes that the inefficient news communication value presents the characteristics of individuality and fragmentation in the construction reality, which may erode the value foundation of the social community and is not conducive to the formation of a social consensus. Algorithmic news production lacks a public opinion guidance mechanism and impacts the mainstream media's public opinion guidance, so it is difficult to form a social consensus. The algorithm can accurately know the user's reading preference, but the algorithm cannot further judge the public value of news and cannot guarantee the comprehensiveness and objectivity of the reality constructed by news.

The "black box problem" of the algorithm is proposed in the literature. Many scholars have also mentioned in their research that deep intelligence and algorithms do have a "technical black box" phenomenon, which is mainly due to the opacity and difficulty of supervising the algorithm process. Some scholars worry that this will have a bad impact on the news production pattern. For example, "flow" becomes an important reference for editors' choice, highquality content is cold, and it becomes difficult to review the authenticity of news [23].

There is no doubt that algorithm recommendation technology plays an important role in news dissemination that cannot be ignored [24]. For example, improving the accuracy, effectiveness, and pertinence of news dissemination is an optimized solution tool in the attention economy, which can personalize information. However, the role of algorithms should not be overstated. The introduction of algorithm technology into the field of news dissemination has a great impact on the information dissemination pattern. Its research is also constantly deepening. The current news value evaluation method designed by scholars has a high degree of deep dependence and can process a small amount of data. Therefore, this paper proposes a standard for judging news value and introduces a deep neural network model, hoping to realize the automatic evaluation and batch calculation of news value.

\section{Related Algorithm Principle}

3.1. Deep Neural Network. Neural network [25-27], as the name implies, is to use computer technology to simulate the neural network structure of the human brain. The goal is to realize some basic functions of the human brain, such as learning and memory [24]. Specifically, it is to simulate the neural network of the human brain through mathematical models. An information processing system constructed by the structure and meta-activities of the Scriptures can be described as a combination of research results from multiple disciplines such as modern medicine, psychology, and mathematical logic.

The information processing [28] capability of a deep neural network is mainly determined by two aspects. On the 
contrary, the topology of the network, specifically neurons, determines the input and output characteristics of the network unit, that is, the activation characteristics of the network unit. The structure, as well as the design of the connecting method [29], such as the number of jingling elements, was selected.

It can be seen that the advantage of deep neural networks is that they do not need to inform the exact functional relationship between input and output in advance and do not need to set a large number of parameters. You only need to know what the nonconstant factors that cause the output change are. Figure 1 shows the key steps of removing complex element weights and determining functional relationships.

In general, the following steps must be included in the construction of a deep neural network: The first step is to set up the network, which includes selecting the number of input and output layers as well as the number of neurons [30]. The second step is to design the hidden layer, which includes deciding on the number of layers and neurons, choosing an activation function, determining the initial connection weight, and determining the learning rate. It also includes the method for calculating the output result, the method for calculating the error, and the iterative update of the connection weight, among other things.

3.2. News Value Evaluation Index and Sample Selection. At present, scholars generally believe that the newness of time mainly includes two aspects: the time is recent and the content is new. First of all, the time is short. Li Liangrong believes that the shorter the time from the occurrence of the event to the public report, the higher the news value. Yang Baojun also believes that the shorter the time distance from the occurrence of the fact to the dissemination of factual information, the more conducive to the realization of news value.

Secondly, the content is new. This "new" mainly includes two aspects. On the one hand, it refers to new situations and new information. The newly occurring facts are completely unknown to the recipient of the intervention; on the other hand, it refers to the fact that the affairs have abnormal changes and can be constantly changed [31].

In the calculation of currentness, this research mainly examines two aspects of content, "timeliness" and "freshness." Among them, "timeliness" is examined from the time dimension, emphasizing the time difference between the occurrence of news facts and the spread of news facts. From an operational point of view, in order to calculate this time difference, it is necessary to obtain two key pieces of information, the "news release time (Ta)" and the "news fact occurrence time (Tb)."

Although scholars have disputes about the definition of news value, they still have a relatively unified understanding of the value object of news value as "news facts." For example, the "standard theory" believes that "news value is a measure of whether objective facts can be news," and the value object emphasized is objective facts; the "quality theory" believes that "news value is the facts themselves that arouse people's common interest." The value object emphasized by "quality" is

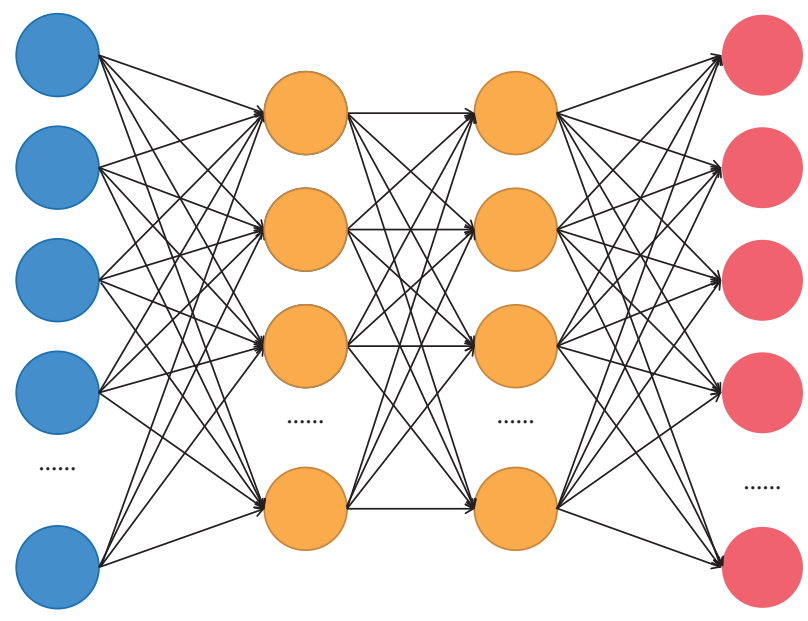

Figure 1: Deep neural network model.

also an objective fact. Importance is an important measure of the impact on the audience and society. If something can affect the vital interests of the broadest masses of the people, it must be very important. Specifically, it mainly includes the following aspects: the number of people affected by the facts, the size of people's interests, the length of time of influence, and the breadth of the influence space.

Significance is mainly to measure the popularity and prominence of various elements that constitute news facts. The discussion on the connotation of saliency currently mainly focuses on the two aspects of the saliency of the subject and the saliency of the event. The so-called saliency of the subject mainly refers to "celebrities" and "famous institutions," that is, "celebrities making news." They either have a high social status or have a high social reputation. Their actions will also be more concerned [32].

Proximity is mainly the various "distances" between news events and news audiences. These "distances" include not only the distance in the sense of geographic space but also the closeness and estrangement at the psychological and emotional level. Geographical "proximity" is because what happens around us is more directly related to our daily lives. Psychological proximity mainly refers to the fact that although the event occurred in a place very far away from us, due to the intertwining or overlapping of economic, cultural, and other factors, these foreign affairs will also arouse public emotional and psychological resonance.

The fun of news is mainly what we usually call Gonzo, which can arouse the curiosity and interest of the audience. Western reporters have always paid more attention to the fun of news, because, in the fierce competition of popular newspapers and periodicals, fun news can help newspapers attract more readers.

\section{Algorithm Modeling Based on Deep Neural Network Model}

4.1. News Text Selection Based on Deep Neural Network. The feature extraction of text is to extract important feature words from the text which represent the meaning of the text 
for quantification, scientific abstraction, and establishment of a mathematical model to replace the meaning of the text. At present, the general practice is to use the vector space model to describe the text, but because some texts may be very long, assuming that the results of the word segmentation are used directly for text vectorization, the dimension of the text vector will have a direct relationship with the length of the text. If the dimension is too large, it may have a negative impact on the time complexity, space complexity, and accuracy of analysis results of subsequent analysis work.

After word segmentation of the news text data, it is necessary to filter some words or words that are meaningless to text analysis in order to save storage space and improve text analysis accuracy. There are two types of stop words that are commonly used. One type is function words, which are words that are universal in human language but have no specific meaning, such as this, yes, and give. The other is a type for modal particles, adverbs, prepositions, conjunctions, and other words with no obvious meaning, such as ZAI DE. Filtering through the stop words list can effectively improve the efficiency and accuracy of text analysis by constructing a stop words list based on the word segmentation results, which includes stop words and punctuation marks.

As a result, it is necessary to purify and reduce the dimensionality of the text vector in order to preserve the text's meaning. The following are the four most commonly used text feature extraction methods: Let us say that $T$ stands for a specific term, Ci stands for a specific category, and $D$ stands for the total number of texts.

Document frequency refers to counting the number of documents in which term $T$ appears in the sample. The calculation formula is as follows:

$$
D G(T)=\sum_{i=1}^{D} P(T, C)
$$

The basic idea is as follows: Calculate the DF value of each entry $T$ with respect to the category $\mathrm{Ci}$, and then compare it with a preset threshold. If it is greater than the threshold, entry $T$ is selected as a feature word; otherwise, the option $T$ is chosen.

The advantage of the document frequency method is that the principle is simple and easy to implement, and the amount of calculation is small. However, the algorithm believes that high-frequency words contribute more to text classification or text content expression than low-frequency words. Therefore, some important feature words with low frequency but text keywords cannot be extracted, which will affect the accuracy of text analysis.

The size of the mutual information value is positively correlated with the degree of correlation between the entry and the category, and its formula is as follows:

$$
M I(T, C)=\log \left(\frac{P\left(T \mid C_{i}\right)}{P(T)}\right) .
$$

The CHI algorithm's basic idea is to compare the actual value and the theoretical value under hypothetical conditions to see if the hypothesis is valid based on the deviation. In text classification, for example, we must determine whether a word $A$ and a category $C$ are independent of one another. If they are unrelated, we will not be able to tell whether the corresponding news belongs to category $C$ based on the appearance of word $A$. Then look to see if $A$ has nothing to do with category $C$ in the sample. The hypothesis is valid if the deviation is small (within a certain range); otherwise, the hypothesis is invalid, indicating that the two variables are not related. But, unlike the traditional chisquare test, we do not need to set an artificial threshold to measure the degree to which word $A$ is related to category $C$ in order to use word $A$ as a feature word of category $C$. As a result, we will use the difference measurement method to determine which is the most relevant. The difference measurement formula is shown in formula (3), assuming that the theoretical value is $E$ and the actual value is $X$ :

$$
\text { Deviation }=\sum_{i=1}^{n} \frac{\left(X_{i}-E\right)^{2}}{E} .
$$

Suppose that there are $N$ pieces of news, of which $M$ pieces belong to the sports category. Table 1 gives statistics on the attribution of the characteristic word "missile" and the category "military."

Assuming that the word "missile" and the category "military" are independent of each other, then "missile" should appear in all texts with equal probability, and its probability is shown in the following equation:

$$
P=\frac{A+B}{N} \text {. }
$$

Therefore, the text number of missiles in the military category is shown in the following formula:

$$
E_{11}=\frac{A+B}{N}(A+C) \text {. }
$$

The actual value is $A$, so the degree of deviation is shown in the following equation:

$$
D_{11}=\frac{(A+B)^{2}}{E_{11}} .
$$

In the same way, the deviation degree D12 of the missile that does not belong to the military category but the word appears, the deviation degree D21 of the missile that belongs to the military category but does not contain the word, and the deviation degree D22 of the missile that does not belong to the military category and the word does not appear can be obtained. Therefore, the chi-square test value of feature word $A$ "missile" and category $C$ "military" can be obtained as shown in the following equation:

$$
\begin{aligned}
x^{2}(A, C) & =D_{11}+D_{12}+D_{21}+D_{22} \\
& =\frac{N(A D-B C)^{2}}{(A+C)(A+B)(B+D)(C+D)} .
\end{aligned}
$$

In practical applications, $N$ is the total number of texts in a given training set, which is a constant. For a category $C$, 
TABle 1: Statistics on the attribution of the characteristic word "missile" and the category "military."

\begin{tabular}{lccc}
\hline Feature word selection & Belong to military & Not military & Total text \\
\hline Including "missile" & $A$ & $B$ & $A+B$ \\
Does not include "missile" & $C$ & $D$ & $C+D$ \\
Total text & $A+C$ & $B+D$ & $N$ \\
\hline
\end{tabular}

Obviously, $A+B+C+D=N ; A+C$ represents the number of texts belonging to sports, and then $A+C=M$, so $B+D=N-M$.

$A+C$ and $B+D$ are the same value for each word, so for a given sample set and category, the chi-square test value can save the calculation of these values. As a result of the chisquare, the formula for calculating the test value can be improved as indicated in the following:

$$
x^{2}(A, C)=\frac{\left(A D-B C^{2}\right)}{(A+B)(C+D)} .
$$

4.2. Text Generalized Process Based on Deep Neural Network Model. The general process of text classification can be divided into two parts. The first part is the training process of text classification, also known as the construction process of the classification model, and the second part is the process of classifying unknown text. In the first part, we need to take real and correct news texts and corresponding categories and then perform word segmentation, word frequency statistics, feature word extraction, text vectorization, and other operations on the news data. After converting the data format required by the classifier, apply classification algorithms (e.g., Bayes, support vector machine, neural network, etc.) to learn the training samples to obtain a classification model. The second step is to apply the training results of the first step to calculate the new news text data and output the category results. The flow chart is shown in Figure 2.

The core idea is to use a set of sample sets of known categories as the training set and obtain a mapping relationship from input feature data to output category data through certain algorithm learning, feedback, and training. It has the three following characteristics.

It is specified that $A 1, A 2, \ldots, A n$ are $n$ features of the data set, $\{C 1, C 2, \ldots, C m\}, C=$ is $m$ categories, and an example sample is $X=\{x 1, x 2, \ldots, x n\}$, where $x i$ represents the value of feature $A i$ corresponding to sample $X$, where $c(X)$ represents the class label obtained by classification, and $P(X \mid C i)$ represents the posterior probability of sample $X$ belonging to category $\mathrm{C} i$. However, it is difficult to calculate the posterior probability, so it is assumed that all features are independent of each other in the deep neural network classifier, as shown in the following equation:

$$
P\left(A_{i} \mid C\right), A_{j}=P\left(A_{i} \mid C\right), \forall A_{i}, A_{j}, P(C)>0 .
$$

This is graphically represented as shown in Figure 3.

\section{Analysis of the Experimental Results of the Value of News Communication}

The experimental data in this article was manually collected in 2016 to test the performance and accuracy of the value evaluation detection scheme in this article, including the "Nepal Earthquake," "Oriental Star Shipwreck," and "Two-Child Policy." There are 101 reports in total, covering five hot topics. Each hot topic's news is recorded as a group of news with high news communication value. The data in the experiment are all the news that are judged to report the same event after manual reading in order to ensure the experiment's accuracy. If the news in a group is judged as news dissemination value news in the following experiment, we believe that the value evaluation test is correct. We believe that the value evaluation test is incorrect if the news in a group is detected as nonnews dissemination value news. Figures 4-8 depict the outcomes.

Based on the foregoing, it can be concluded that when the number of feature words extracted is $40 \%$ of the total number of text words, each index is the best, and they are all higher than other indexes. When the percentage of feature terms is less than $40 \%$, the poor index is due to the fact that there are fewer feature words, which cannot fully express the text's core idea, so the news communication value calculation result is poor; when the percentage of feature terms is greater than $40 \%$, the poor index is due to the fact that there are more feature words, which can fully express the text's core idea, so the news communication value calculation result is poor. When a large number of feature words are extracted, there will be some words that have no special meaning and cannot represent the text's unique thoughts as feature words, causing the vector's noise to be high, lowering the various indicators for calculating the value of news communication. As a result, the percentage of feature terms extracted in this experiment is set at $40 \%$.

Any two articles will get a news communication value measurement value through the news communication value calculation method introduced in this article. This article sets different thresholds of news communication value and compares the news communication value of a given sample data set under different thresholds.

Figures 9 and 10 are the analysis and comparison of the experimental results of the evaluation and detection of news text news communication value under the three evaluation indicators of correctness rate, recall rate, and F1 measurement value with different thresholds. THe following can be seen from the figure:

(1) When the threshold value continues to increase, the correct rate of the calculation of the value of news communication also increases. When the threshold value is 0.9 , it reaches $100 \%$.

(2) When the threshold is 0.6 , the recall rate calculated by the evaluation of news communication value is the highest, which is $75.3 \%$. 


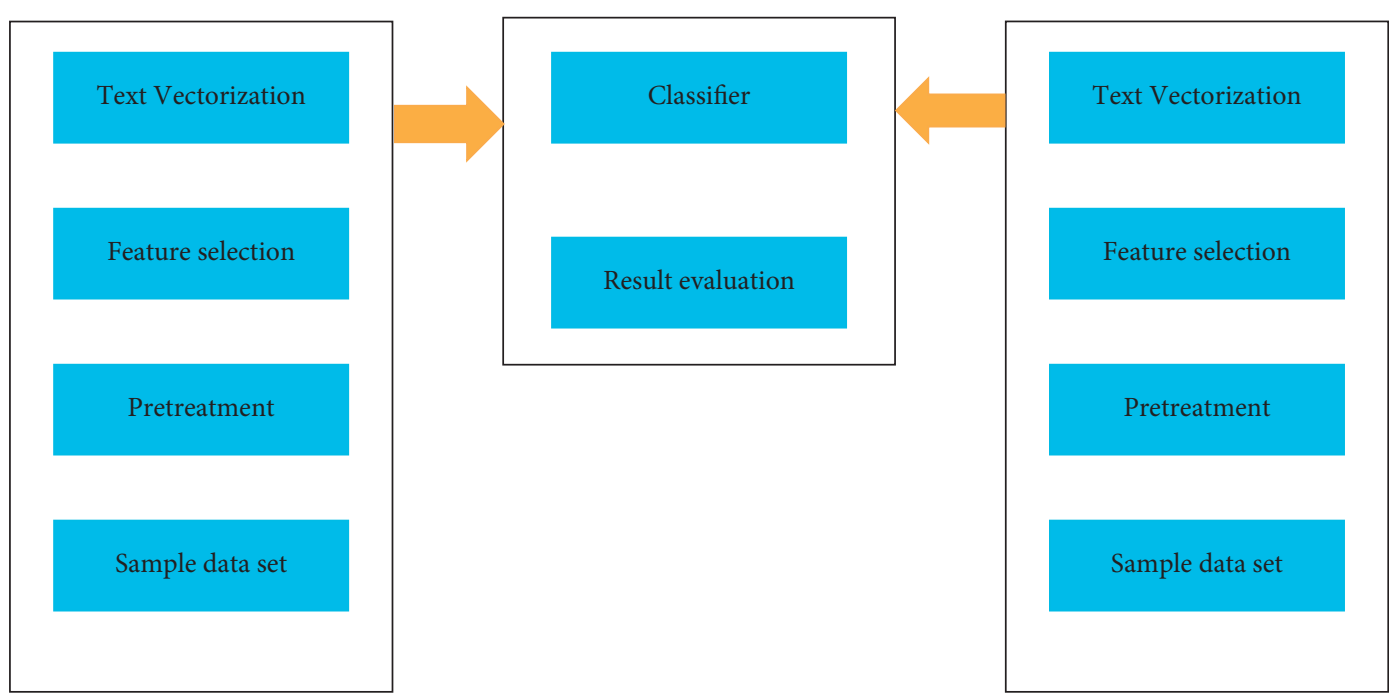

Figure 2: General flow chart of text classification.

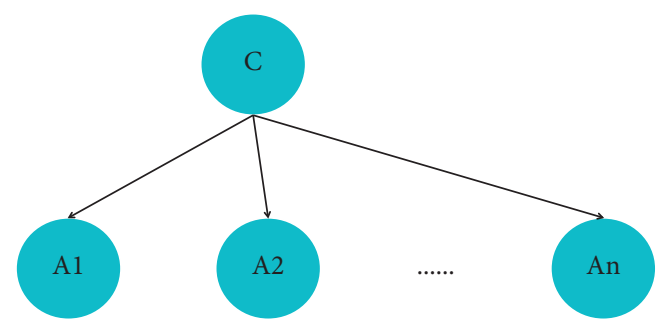

FIGURE 3: Deep neural network model classifier.

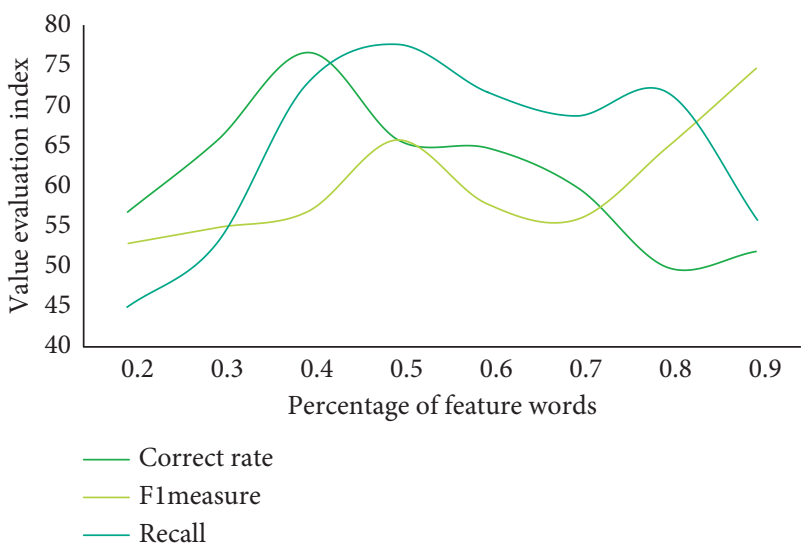

Figure 4: The value evaluation index curve of different percentages of characteristic words.

(3) When the threshold value is 0.6 , the F1 measurement value calculated by the news communication value evaluation is the highest, which is $79.8 \%$.

In summary, the threshold is 0.6 , and each indicator is the best case, and they are all 4 to 5 percentage points higher than other indicators. When the threshold is set too low, the system will classify texts with a low calculated value of news dissemination value evaluation and irrelevant content as similar news; when the threshold is set too high, the system will classify texts with a calculated value of news

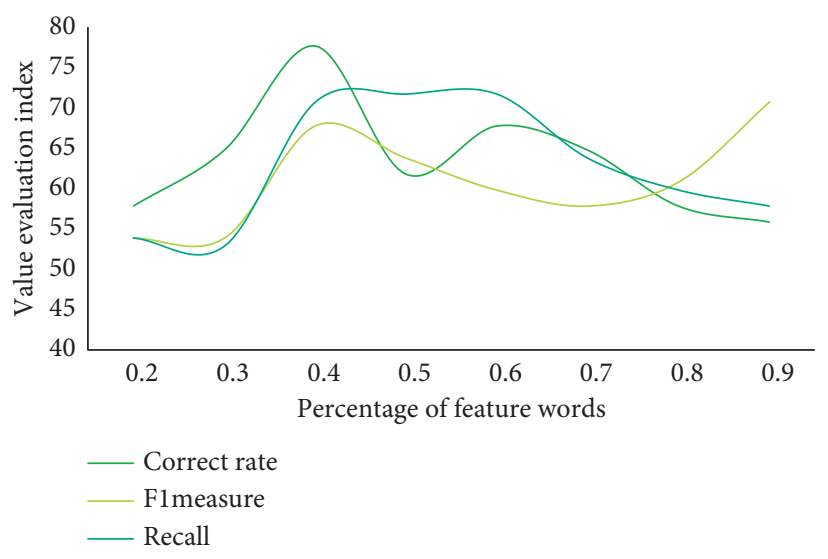

FIgURE 5: The value evaluation index curve of different percentages of characteristic words.

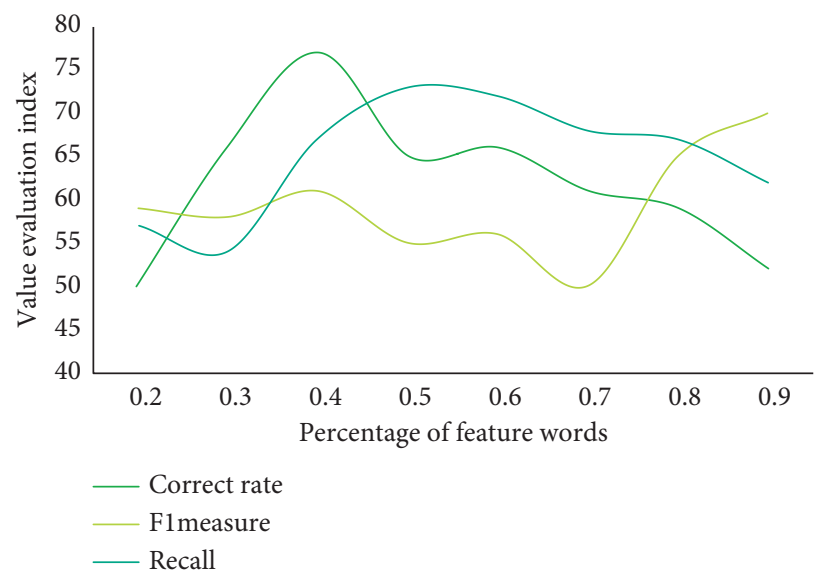

FIgURE 6: The value evaluation index curve of different percentages of characteristic words.

dissemination value less than the set threshold as similar news; and when the threshold is set too high, the system will classify texts with a calculated value of news dissemination 


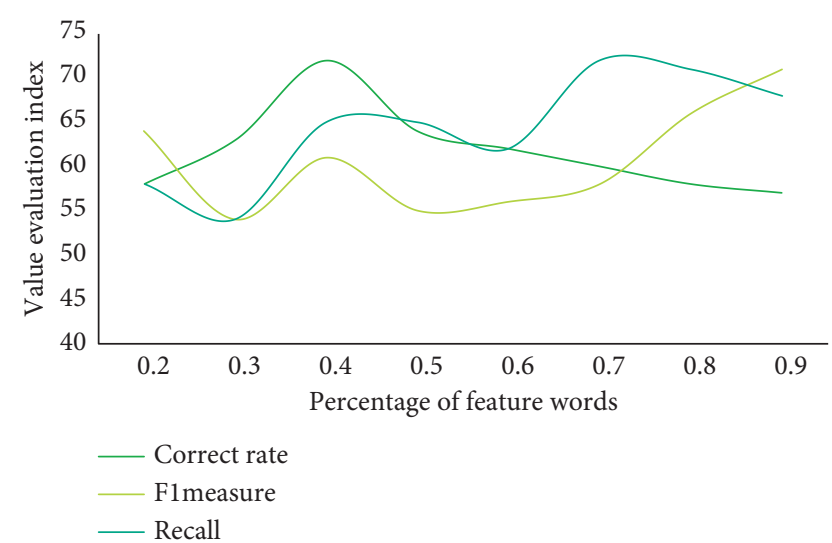

Figure 7: The value evaluation index curve of different percentages of characteristic words.

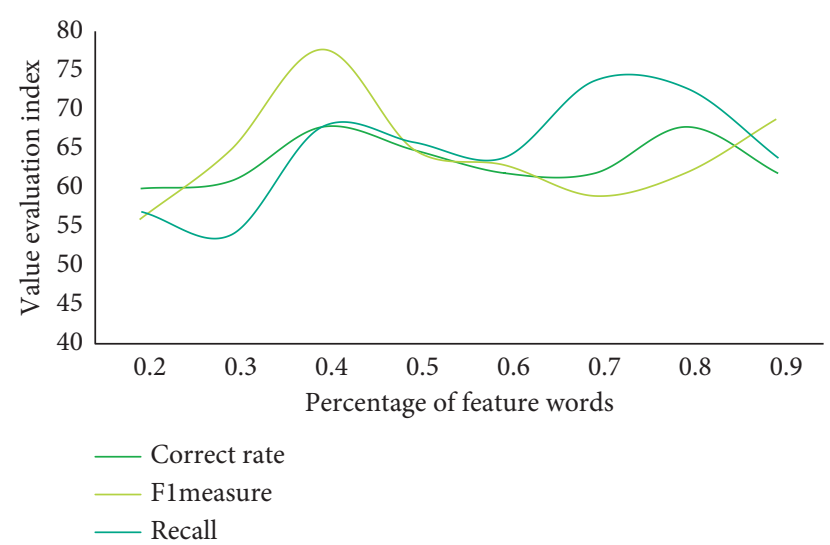

Figure 8: The value evaluation index curve of different percentages of characteristic words.

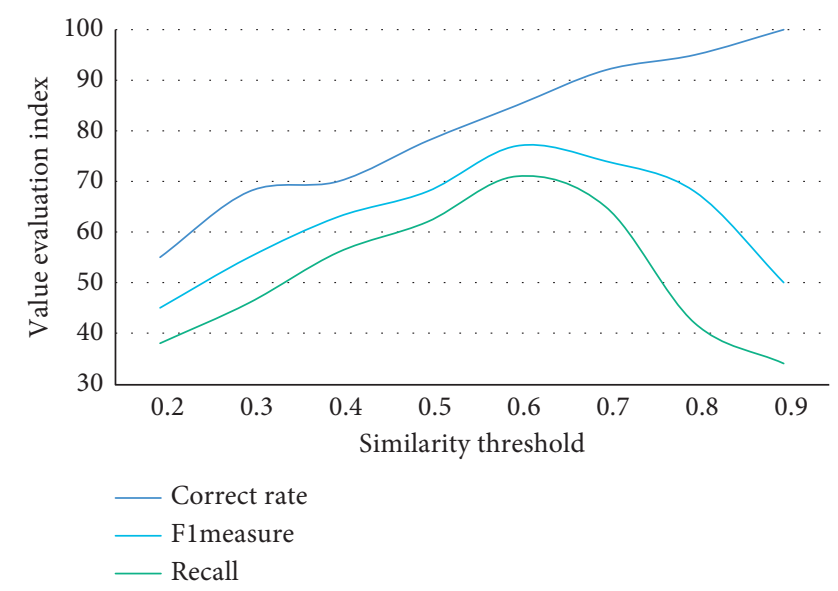

Figure 9: Curves of the accuracy of the calculation of the value of text news communication with different thresholds.

value less than the set threshold as similar news. When the threshold is set too high or too low, original texts with similar content cannot be classified as similar news, so the correct rate is lower. As a result, the highest point 0.6 of the F1 metric value transition is chosen as the threshold for judging whether the texts are similar in this experiment.

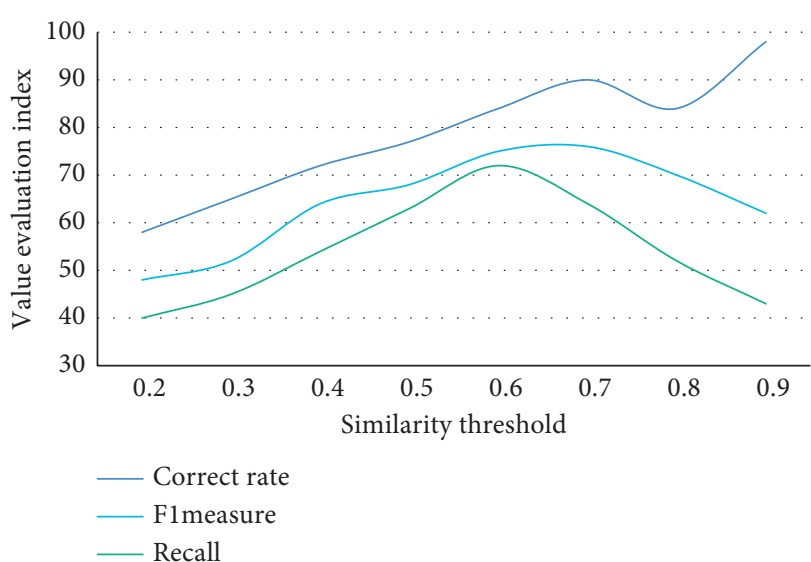

FIgURE 10: Curves of the accuracy of the calculation of the value of text news communication with different thresholds.

Based on the experimental data of the percentage of feature words and the evaluation threshold of news communication value, when we select the optimal percentage of $40 \%$ and the threshold value of 0.6 , the accuracy rate of news communication value evaluation calculated based on the experimental sample data can reach $85.7 \%$. The recall rate reached $73.1 \%$, and the $\mathrm{F} 1$ measurement value reached $78.9 \%$, so we can think that the experiment can be more accurate for the calculation of the value of news text news communication.

\section{Conclusions}

This paper builds a set of news evaluation index systems and a set of algorithm evaluation models of news value based on deep neural networks and news value as the theory. The final result output is completed through the learning and training of the training set. Scholars have become increasingly interested in the intersection of social sciences and computer sciences in recent years. Text mining and other natural language processing technologies are increasingly being used in the social sciences, and this trend is continuing. How to use big data text analysis technology to mine valuable information and knowledge from massive news texts in the field of journalism and communication and how to use artificial intelligence technology to realize effective news dissemination and improve users' news information acquisition efficiency represent a topic that scholars are interested in, and they are also the focus of this study.

Although there is a well-developed judgement framework for judging news value in the social sciences, the implementation of specific evaluation work not only places high demands on the professional quality of the evaluator but also requires a significant amount of manual labour. As a result, nearly $60 \%$ of the news value scores were correct, and news with a 1 point prediction error accounted for more than $80 \%$ of the total number of samples. Such error data also demonstrates that the model is capable of automatically calculating news value, implying that the machine can learn human value judgments and has a certain degree of judgement stability. However, the author must acknowledge 
that this article is merely a first attempt. There have been some basic investigations into the field of news value evaluation research, but there are many flaws and there is room for improvement.

\section{Data Availability}

The data used to support the findings of this study are included within the article.

\section{Conflicts of Interest}

The author declares that there are no conflicts of interest.

\section{Acknowledgments}

This study was supported by the Fundamental Research Funds of Heilongjiang Education Department (Grant No. 2018-KYYWF-0974).

\section{References}

[1] M. Cao, H. Li, and R. Zhao, "A pitch detection algorithm based on deep neural network," Microelectronics and Computer, vol. 33, no. 6, pp. 143-146, 2016.

[2] T. Hu, H. Wang, and W. Yin, "Multi-label news classification algorithm based on deep two-way classifier chain," Journal of Zhejiang University: Engineering Edition, vol. 53, no. 11, p. 8, 2019.

[3] S. Cai, C. L Xu, and Q. Gao, "Particle image velocity measurement algorithm based on deep neural network," Acta Aerodynamics, vol. 37, no. 3, pp. 455-461, 2019.

[4] S. Cai, C. Xu, and Q. Gao, "Particle image velocimetry algorithm based on deep neural network," Acta Aerodynamics, vol. 37, no. 3, p. 7, 2019.

[5] C. Zhong, C. Peng, and W. Du, "Research on red blood cell recognition algorithm based on deep convolutional neural network," Wireless Internet Technology, vol. 17, no. 5, pp. 23-24+39, 2020.

[6] J. Huang and G. Zhang, "Overview of target detection algorithms for deep convolutional neural networks," Computer Engineering and Applications, vol. 56, no. 17, p. 12, 2020.

[7] G. Zhang, "Research on point cloud recognition algorithm based on deep neural network," Industrial Control Computer, vol. 32, no. 8, pp. 121-122, 2019.

[8] C. Zhang and Z. Huang, "An effective vehicle retrieval algorithm based on convolutional neural network," Technology Innovation and Application, vol. 13, pp. 6-9, 2021.

[9] H. Pei, "Research on deep neural network learning based on improved BP algorithm," Mechanical Strength, vol. 40, no. 4, p. 6, 2018.

[10] L. Yao, J. Zhu, and Z. Ma, "Single-channel speech separation algorithm based on deep neural network," Journal of Information Technology, vol. 42, no. 7, p. 4, 2018.

[11] C. Wang, X. Tang, and S. Gao, "Infrared scene understanding algorithm based on deep convolutional neural network," Infrared Technology, vol. 39, no. 8, p. 6, 2017.

[12] J. Liu, "A review of deep learning algorithms based on artificial neural networks," China New Communications, vol. 20, no. 6, pp. 193-194, 2018.

[13] J. Yang, Q. Wang, and X. Xiaohua, "Lung nodule detection model based on deep convolutional neural network algorithm," Mathematical Modeling and Applications, no. 4, pp. 1-9, 2017.

[14] L. Cheng and M. Gao, "Recommendation algorithm based on deep neural network," Modern Computer (Professional Edition), vol. 622, no. 22, pp. 5-9, 2018.

[15] L. Ma, "Research on handwriting deep learning algorithm based on convolutional neural network," Computer Science and Application, vol. 8, no. 11, p. 9, 2018.

[16] Y. Cheng, "Research on car model recognition algorithm based on multi-column deep convolutional neural network," Journal of Changchun Normal University, vol. 39, no. 6, p. 5, 2020.

[17] S. Zhang, "Progress of target detection algorithms based on deep convolutional neural networks," Journal of Nanjing University of Posts and Telecommunications: Natural Science Edition, vol. 39, no. 5, p. 9, 2019.

[18] Y. Wang, Y. Zhuo, and Y. Wu, "Image fragmented information question answering algorithm based on deep neural network," Computer Research and Development, vol. 55, no. 12, pp. 18-28, 2018.

[19] J. Xue, X. Gu, and T. Ni, "Auto-weighted multi-view discriminative metric learning method with Fisher discriminative and global structure constraints for epilepsy EEG signal classification," Frontiers in Neuroscience, vol. 14, Article ID 586149, 2020.

[20] T. Ni, Y. Ding, J. Xue, K. Xia, X. Gu, and Y. Jiang, "Local constraint and label embedding multi-layer dictionary learning for sperm head classification," ACM Transactions on Multimedia Computing, Communications, and Applications, vol. 17, no. 3s, pp. 1-16, 2021.

[21] Y.-Y. Zheng, J.-L. Kong, X.-B. Jin, X.-Y. Wang, M. Zuo, and M. Zuo, "CropDeep: the crop vision dataset for deeplearning-based classification and detection in precision agriculture," Sensors, vol. 19, no. 5, p. 1058, 2019.

[22] Q. Guo, X. Lu, and Y. Xie, "Efficient visual target tracking algorithm based on deep spectrum convolutional neural network," Infrared and Laser Engineering, vol. 47, no. 6, p. 6, 2018.

[23] X. Xiong, X. Chen, and Y. Wu, "Fall detection algorithm based on improved recurrent neural network deep learning," Computer Programming Skills and Maintenance, vol. 139, no. 3, pp. 126-127, 2020.

[24] Y. Wu and Y. Xu, "An algorithm for detecting premature ventricular beats based on an improved deep convolutional neural network," Computer Applications and Software, vol. 36, no. 11, p. $5,2019$.

[25] Z. Wu and W. Chu, "Sampling strategy analysis of machine learning models for energy consumption prediction," in Proceedings of the 2021 IEEE 9th International Conference on Smart Energy Grid Engineering (SEGE), August 2021.

[26] M. Zhao, "Faster Mean-shift: GPU-accelerated clustering for cosine embedding-based cell segmentation and tracking," Medical Image Analysis, vol. 71, Article ID 102048, 2021.

[27] J. Kong, H. Wang, X. Wang, X. Jin, X. Fang, and S. Lin, "Multi-stream hybrid architecture based on cross-level fusion strategy for fine-grained crop species recognition in precision agriculture," Computers and Electronics in Agriculture, vol. 185, Article ID 106134, 2021.

[28] Z. Huang, Y. Zhang, Q. Li, T. Zhang, N. Sang, and H. Hong, "Progressive dual-domain filter for enhancing and denoising optical remote-sensing images," IEEE Geoscience and Remote Sensing Letters, vol. 15, no. 5, pp. 759-763, 2018.

[29] Y. Chen, "Algorithm improvement based on deep convolutional neural network," Electronics World, vol. 541, no. 07, pp. 59-60, 2018. 
[30] H. Li and M. Zhu, "Small target detection algorithm based on deep convolutional neural network," Computer Engineering and Science, vol. 42, no. 4, pp. 649-657, 2020.

[31] Y. Huang, X. Duan, and S. Sun, "Research on deep neural network training algorithm based on improved sigmoid activation function," Computer Measurement and Control, vol. 2, pp. 126-129, 2017.

[32] Y. Chen and D. Sun, "Target detection algorithm based on CS optimized deep learning convolutional neural network," Machine Tool \& Hydraulics, vol. 48, no. 6, p. 6, 2020. 